\title{
GENETICS
}

\section{RNA topoisomerase involved in neurodevelopmental disorders}

Two independent lines of research have discovered that TOP $3 \beta$ protein is an mRNA topoisomerase involved in schizophrenia, fragile X syndrome and cognitive impairment. Altered mRNA metabolism has been previously implicated in neurocognitive disorders, but the exact pathogenetic mechanisms have proven elusive.

\section{This discovery is most exciting to us because it should open up a whole new field... 77}

In most populations, the genetic variants that contribute to neurodevelopmental disorders are so rare that getting statistical evidence of their involvement is difficult. An international collaboration headed by Nelson Freimer, Aarno Palotie and Utz Fischer, therefore, studied a Finnish subpopulation that has a high risk of neurodevelopmental disorders. The group report that schizophrenia and cognitive difficulties in this population are both associated with a deletion in chromosomal region 22q11.22more specifically, in $T O P 3 B$, the gene encoding TOP3 $\beta$.

TOP $3 \beta$ is a topoisomerase $-\mathrm{a}$ molecule able to transiently cleave and rejoin nucleic acid strands to resolve topological stress. Freimer and colleagues discovered that instead of DNA, the substrate of TOP $3 \beta$ was RNA. Their findings were independently confirmed in a second study by Weidong Wang and colleagues who demonstrated that TOP $3 \beta$ can catalyze a complete topoisomerase reaction on RNA, making it the first eukaryotic RNA topoisomerase to be discovered. "This discovery is most exciting to us because it should open up a whole new field," says Wang.

Through immunoprecipitation studies, both groups also found that
TOP3 $\beta$ forms a trimeric complex that is recruited to mRNAs. Interestingly, this complex contains the fragile X mental retardation protein (FMRP), a known regulator of neuronal mRNA translation. Wang and collaborators conducted knockout studies in Drosophila melanogaster and mice, which demonstrated that TOP $3 \beta$ and FMRP co-regulate synapse formation and neurodevelopment.

The findings suggest that TOP3 $\beta$ is involved in the pathophysiology of several neurodevelopmental disorders. For example, a mutation in FMRP that prevents its direct and indirect interaction with other proteins, including TOP $3 \beta$, leads to fragile $\mathrm{X}$ syndrome. FMRP also regulates the translation of proteins implicated in autism spectrum disorders. Wang hopes that in the future, drugs that target TOP $3 \beta$ activity could be used as therapeutic interventions for various mental disorders.

The findings could provide new insight into pathogenesis of a diverse range of neurocognitive disorders. "Biochemical studies suggest a link between schizophrenia and fragile X syndrome, two disorders usually considered entirely distinct from one another," notes Freimer. He goes on to contend that the association of the same deletion in TOP3 $\beta$ with both schizophrenia and cognitive impairment supports the notion that the biology underlying common brain and behavioural disorders is unlikely to correspond directly to traditional diagnostic categories.

Hemi Malkki

Original articles Stoll, G. et al. Deletion of TOP3 $\beta$, a component of FMRP-containing mRNPs, contributes to neurodevelopmental disorders. Nat. Neurosci. doi:10.1038/nn.3484 | Xu, D. et al. Top3 $\beta$ is an RNA topoisomerase that works with fragile $X$ syndrome protein to promote synapse formation. Nat. Neurosci. doi:10.1038/ nn.3479 